\title{
Prostatic Adenocarcinoma With Pseudocapsule
}

\author{
Yuichi Sato ${ }^{a}$, Toshiki Oguro, ${ }^{a, c}$, Shin Kumagai ${ }^{a}$, Soichiro Ogawa ${ }^{a}$, Norio Takahashia, \\ Nobuhiro Haga $^{\mathrm{a}}$, Michiko Nakano ${ }^{\mathrm{b}}$, Kei Ishibashia ${ }^{\mathrm{a}}$, Ken Aikawa ${ }^{\mathrm{a}}$, Yoshiyuki Kojima ${ }^{\mathrm{a}}$
}

\begin{abstract}
A 72-year-old man was referred to our hospital for treatment of prostatic adenocarcinoma with atypical imaging findings. The periprostatic mass was distinguishable from the prostate on magnetic resonance imaging, although the prostatic adenocarcinoma had been identified by needle biopsy at a previous hospital. To confirm the site of prostatic adenocarcinoma, transrectal ultrasonographydirected biopsy of the prostate and solitary mass was performed in our hospital. Diagnosis by re-biopsy revealed prostatic adenocarcinoma existing as a solitary mass. Radical prostatectomy was performed with no evidence of metastasis on imaging findings. Intraoperative findings revealed no adhesion between the prostate and pelvic floor. Pathological diagnosis revealed negative surgical margins, and a thin crust surrounding the adenocarcinoma. Strict pathological examination indicated a tiny adenocarcinoma existing within morphologically normal prostate tissue comprising nearly the entire mass. Based on these results, the final pathological diagnosis was locally advanced adenocarcinoma with pseudocapsule. In the present case, we concluded that a tiny prostatic adenocarcinoma had expanded in the lateral pelvic fascia, which formed a pseudocapsule. By 14 months postoperatively, prostate-specific antigen levels had decreased below the limit of detection. To the best of our knowledge, prostatic adenocarcinoma with pseudocapsule has not been described previously.
\end{abstract}

Keywords: Prostatic adenocarcinoma; Pseudocapsule; Atypical imaging findings

\section{Introduction}

A variety of uncommon masses that can arise from the pel-

\footnotetext{
Manuscript accepted for publication May 13, 2013

${ }^{\mathrm{a}}$ Department of Urology, Fukushima Medical University, Fukushima, Japan

${ }^{b}$ Department of Urology, Hanawa Kosei Hospital, Fukushima, Japan ${ }^{\mathrm{c}}$ Corresponding author: Toshiki Oguro, 1 Hikarigaoka, Fukushima City, Fukushima 960-1295, Japan. Email:oguro10@yahoo.co.jp
}

doi: http://dx.doi.org/10.4021/wjnu76w vis are increasingly being encountered because of the growing use of cross-sectional imaging, particularly computed tomography (CT) and magnetic resonance imaging (MRI). Accurate diagnosis and appropriate treatment of such masses is clearly important. Prostatic adenocarcinoma is a common disease in the urological fields. Imaging findings of patients with advanced disease are often of atypical prostate images, with irregularities of the prostate capsule and seminal vesicle. Occasionally, prostate cancer invades surrounding tissue, such as the bladder, pelvic floor muscle, and rectum. Advanced prostatic cancer is relatively easily diagnosed by high levels of prostate-specific antigen (PSA). However, diagnosis of localized prostatic adenocarcinoma with atypical imaging findings is difficult. In addition, prostatic adenocarcinoma with pseudocapsule does not seem to have been described previously, making such cases more difficult to accurately diagnose. This represents the first report of prostatic cancer with pseudocapsule cured by radical prostatectomy.

\section{Case Report}

A 72-year-old man was referred to our hospital for treatment of prostatic cancer with atypical imaging findings. PSA level was elevated, at $11.3 \mathrm{ng} / \mathrm{mL}$. Digital rectal examination revealed a non-movable nodule palpable at the left base of the prostate. Transrectal ultrasonography (TRUS) revealed an isoechoic mass above the prostate, and MRI showed a solitary mass with circumferential capsule above the prostate (Fig. 1). Although prostatic cancer had been diagnosed in the previous hospital based on needle biopsy, whether the prostatic cancer had developed in the mass or within the normal morphology of the prostate remained unclear. We recommended re-biopsy with the intent of confirming the site of prostatic cancer. TRUS-directed biopsy of the prostate and mass demonstrated adenocarcinoma only in the mass, and this adenocarcinoma showed positive results for PSA on immunohistochemical examination. No metastases were identified on $\mathrm{CT}$ or bone scintigraphy. Radical prostatectomy and pelvic lymphadenectomy were performed 2 months after diagnosis of prostatic carcinoma. The postoperative course has been uneventful and the patient is currently doing well, 


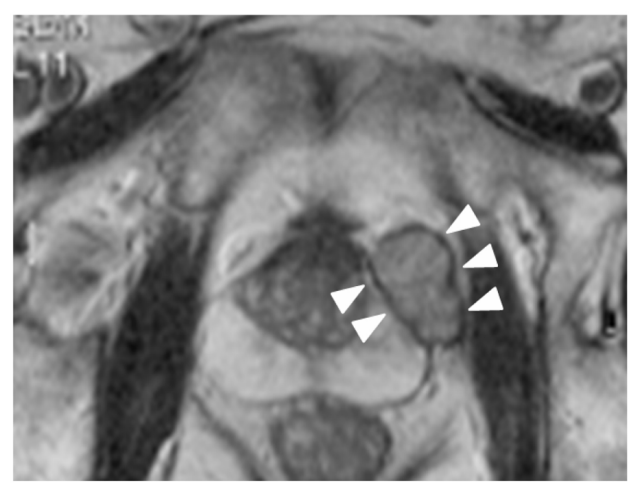

Figure 1. T2-weighted MRI, axial plane. A small, round encapsulated mass is apparent above the prostate. The mass is distinguishable from the prostate.

with no metastatic disease. As of 14 months postoperatively, PSA level has decreased below the level of detection $(<0.02$ $\mathrm{ng} / \mathrm{mL})$.

\section{Pathological examination}

The prostate and tumor together weighed $19.0 \mathrm{~g}$. Tumor with necrosis was present on the left side of the prostate. The tumor measured $2.2 \times 2.4 \times 2.1 \mathrm{~cm}$ and was surrounded by a thin crust. Microscopic examination revealed a tiny prostate cancer existing within morphologically normal prostate tissue, and all surgical margins were negative. The tumor was assigned a Gleason score of $3+4$. All excised lymphatic tissue was negative for carcinoma (Fig. 2). Although we initially supposed that the adenocarcinoma was growing within ectopic prostatic tissue (EPT) in the periprostatic fascia, the present case had a different pathological finding about the tumor, as no benign gland was present. This microscopic finding differed from previous reports, which have uniformly described the presence of both benign and malignant glands within the tumor. However, we had difficulty detecting this tiny prostate cancer infiltrating the prostatic fascia, and demonstrating the presence of growth between the capsule of the prostate and the lateral pelvic fascia (LPF). Because the tumor had no benign glands and only a tiny prostate cancer existing near the tumor, the pathological diagnosis was adenocarcinoma growing between layers of periprostatic fascia acting as a pseudocapsule.

\section{Discussion}

The prostate has been described as being surrounded by two layers of periprostatic fascia: an inner layer around the prostate termed the 'prostatic capsule'; and an outer layer termed the LPF $[1,2]$. However, while the prostate has no capsule as such, strict histological examination has revealed prostatic fascia comprising membranous structures in multiple layers, including collagen tissue, adipose tissue, and areolar tissue [3]. Thickness of the LPF varies widely between individuals. Histological examination in the present case revealed multiple relatively thick layers making up the LPF, embedded in varying amounts of adipose tissue and blood vessels. Therefore, one of the reasons that prostatic carcinoma could not extend beyond the LPF was that it had not extended beyond the prostatic fascia.

The presence of myofibroblasts or stromal cells exhibiting smooth muscle features has been detected in the capsules of neoplasms in several organs, including the liver, thyroid

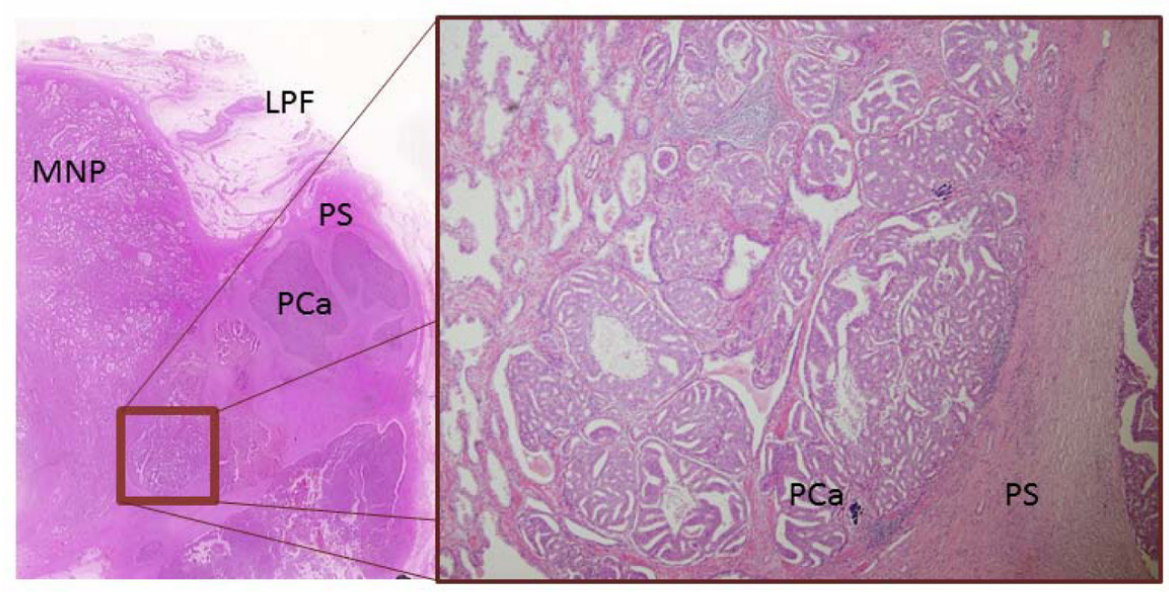

A

B

Figure 2. Pathological examination. A) Histological preparation. The tumor is present in the LPF. A tiny prostate cancer existing near the tumor was pointed out on imaging. B) The tumor is growing between the layers of LPF in a pseudocapsule, comprising a tiny prostate cancer present within morphologically normal prostate tissue. Detecting this tiny prostate cancer infiltrating the prostatic fascia was difficult. 
gland, and pancreas [4-6]. In the urological field, renal cell carcinoma (RCC) is one disease showing encapsulation, and the presence of myofibroblasts and fibrosis has been described in the RCC capsule [7]. In the present case, the "capsule" consisted almost entirely of LPF. Prostate adenocarcinoma with pseudocapsule does not appear to have been reported previously.

Preoperative differential diagnoses for masses in the perivesical or periprostatic soft tissue include some diseases, such as teratoma, prostate cancer arising in EPT, and metastatic neoplasm from the prostate or other organs $[8,9]$. As reaching a definite diagnosis based solely on imaging findings is difficult, histological examination of atypical pelvic masses is necessary.

In the present case, histological examination of a needle biopsy with immunohistochemical staining for PSA revealed the prostate as the origin of the tumor. Teratoma was excluded based on the lack of non-prostatic tissue. In the few reports that have described adenocarcinoma occurring in the EPT $[10,11]$, the presence of both benign and malignant glands has been confirmed. Adams described the first case of adenocarcinoma occurring in the EPT at the inferior prostatic vascular pedicle [10]. Microscopic examination in that case revealed that although the adenocarcinoma was within the normal prostate, the presence of both benign and malignant glands within a nodule separate and distinct from the prostate led to the interpretation that the adenocarcinoma had arisen within EPT, rather than as a metastatic deposit. The lack of metastatic disease in the patient described in that report, even after many years, lends further supportive evidence to this claim. A second case of adenocarcinoma occurring in EPT was described by Gardner [11]. In that case, adenocarcinoma was also present within normal prostatic tissue. At a location distant from the prostate (the dome of the bladder), the presence of both benign and malignant glands confirmed that the tumor had arisen in the EPT. Because the present case included no benign glands, EPT was excluded, and the final diagnosis was prostatic cancer within the LPF. The absence of metastatic disease and the decrease in PSA level to below the limit of detection, maintained even after 1 year, lends further support to this diagnosis. We therefore diagnosed adenocarcinoma growing between the capsule of the prostate and the LPF. Fortunately, infiltration beyond the LPF had not occurred in this case of low-grade prostatic adenocarcinoma with pseudocapsule.

\section{Conclusion}

We have described a case of prostate cancer with pseudocapsule. Our experience with this case suggests that one differential diagnosis for atypical findings on clinical imaging of the prostate is prostate cancer with pseudocapsule. Although no optimal treatment for cases like this has been defined, radical prostatectomy appears to have provided good results in this case.

\section{Disclosure}

The authors have no conflicts of interest to declare.

\section{References}

1. Raychaudhuri B, Cahill D. Pelvic fasciae in urology. Ann R Coll Surg Engl. 2008;90(8):633-637.

2. Tewari A, Peabody JO, Fischer M, Sarle R, Vallancien G, Delmas V, Hassan M, et al. An operative and anatomic study to help in nerve sparing during laparoscopic and robotic radical prostatectomy. Eur Urol. 2003;43(5):444-454.

3. Kiyoshima K, Yokomizo A, Yoshida T, Tomita K, Yonemasu H, Nakamura M, Oda Y, et al. Anatomical features of periprostatic tissue and its surroundings: a histological analysis of 79 radical retropubic prostatectomy specimens. Jpn J Clin Oncol. 2004;34(8):463-468.

4. Ooi LP, Crawford DH, Gotley DC, Clouston AD, Strong RW, Gobe GC, Halliday JW, et al. Evidence that "myofibroblast-like" cells are the cellular source of capsular collagen in hepatocellular carcinoma. J Hepatol. 1997;26(4):798-807.

5. Nakayama H, Enzan H, Miyazaki E, Moriki T, Kiyoku H, Toi M, Kuroda N, et al. High molecular weight caldesmon positive stromal cells in the capsule of thyroid follicular tumours and tumour-like lesions. J Clin Pathol. 2002;55(12):917-920.

6. Kuroda N, Toi M, Nakayama H, Miyazaki E, Yamamoto M, Hayashi Y, Hiroi M, et al. The distribution and role of myofibroblasts and CD34-positive stromal cells in normal pancreas and various pancreatic lesions. Histol Histopathol. 2004;19(1):59-67.

7. Shimasaki N, Kuroda N, Guo L, Jin Y, Miyazaki E, Hayashi Y, Toi M, et al. The participation of myofibroblasts in the capsular formation of human conventional and chromophobe renal cell carcinomas. Histol Histopathol. 2005;20(1):67-73.

8. Yasukawa S, Aoshi H, Takamatsu M. Ectopic prostatic adenoma in retrovesical space. J Urol. 1987;137(5):998-999.

9. Vahidi K, Joe BN, Meng M, Coakley FV, Yeh BM. Review of atypical pelvic masses on CT and MRI: expanding the differential diagnosis. Clin Imaging. 2007;31(6):406-413.

10. Adams JR, Jr. Adenocarcinoma in ectopic prostatic tissue. J Urol. 1993;150(4):1253-1254.

11. Gardner JM, Khurana H, Leach FS, Ayala AG, Zhai J, Ro JY. Adenocarcinoma in ectopic prostatic tissue at dome of bladder: a case report of a patient with urothelial carcinoma of the bladder and adenocarcinoma of the prostate. Arch Pathol Lab Med. 2010;134(9):1271-1275. 